\title{
Research on an alternative LS2P microphone based on a new reciprocity calibration system
}

\author{
Maria Enge ${ }^{1, *}$, Christian Hof $^{2}$ and Salvador Barrera-Figueroa ${ }^{3}$ \\ ${ }^{1}$ SPEKTRA GmbH Dresden, Research and Development, 01189 Dresden, Germany \\ ${ }^{2}$ Federal Institute of Metrology METAS, Acoustic and Vibration, 3003 Bern-Wabern, Switzerland \\ ${ }^{3}$ Danish Fundamental Metrology (DFM) A/S, Acoustic, 2970 Hørsholm, Denmark
}

\begin{abstract}
The microphone calibration by the reciprocity technique specified in IEC 61094-2 is used to determine the sensitivity of laboratory standard microphones according to the IEC 61094-1 with the smallest measurement uncertainty for the use as reference microphones. So far, laboratory standard microphones by the manufacturer Brüel \& Kjær (Type 4160 and Type 4180) are almost exclusively used as laboratory reference microphones. In order to create an alternative, the initiative has been taken to examine the usability of the $1 / 2$-inch laboratory microphones G.R.A.S. $40 \mathrm{AU}-1$. Studies were launched to check the microphone parameters, the stability and the reciprocity of the microphones as well as the compatibility with microphones by Brüel \& Kjær. Basis for the investigation was a new validated reciprocity calibration system. The realization of the system and the research results are presented and discussed. Additionally, results of comparison measurements with national metrology institutes are shown in shortened fashion.
\end{abstract}

\section{Introduction}

The usual primary method of determining the complex pressure sensitivity of laboratory standard (LS) microphones is the pressure reciprocity calibration method (described in the IEC publication 61094-2 [1]). This method takes advantage of the fact that LS microphones are reciprocal transducers. This means that the microphone works both as sound receiver and sound source (transmitter). During calibration, two out of three microphones are coupled acoustically, one of which acts as receiver, the other acting as transmitter. The microphones are connected by a coupler (a small cylindrical cavity). In order to determine the sensitivity of each of the three microphones the electrical transfer impedance of the paired microphones has to be measured precisely. In addition to the electrical transfer impedance, the acoustic transfer impedance of the enclosed air volume between the microphones has to be modelled accurately. The acoustic transfer impedance is influenced by several factors including the microphone parameters.

In the past, this method has been applied almost exclusively to LS microphones manufactured by Brüel \& Kjær - the 1-inch pressure (LS1P) microphone type 4160 and the $1 / 2$-inch pressure (LS2P) microphone type 4180 - and the validity of the approach was

*2maria.enge@spektra-dresden.de 
approved through high effort of metrological investigation and with a large number of microphones.

Currently, comparable research to evaluate alternative types of microphones has not been published as yet. In this report, the results of a first investigation with LS microphones of the type 40AU-1 manufactured by G.R.A.S determined with a new reciprocity calibration system by SPEKTRA are presented.

\section{Design of the reciprocity calibration system}

SPEKTRA developed a new reciprocity calibration system according to the current standard [1]. Figure 1 shows a schematic diagram of the calibration system.

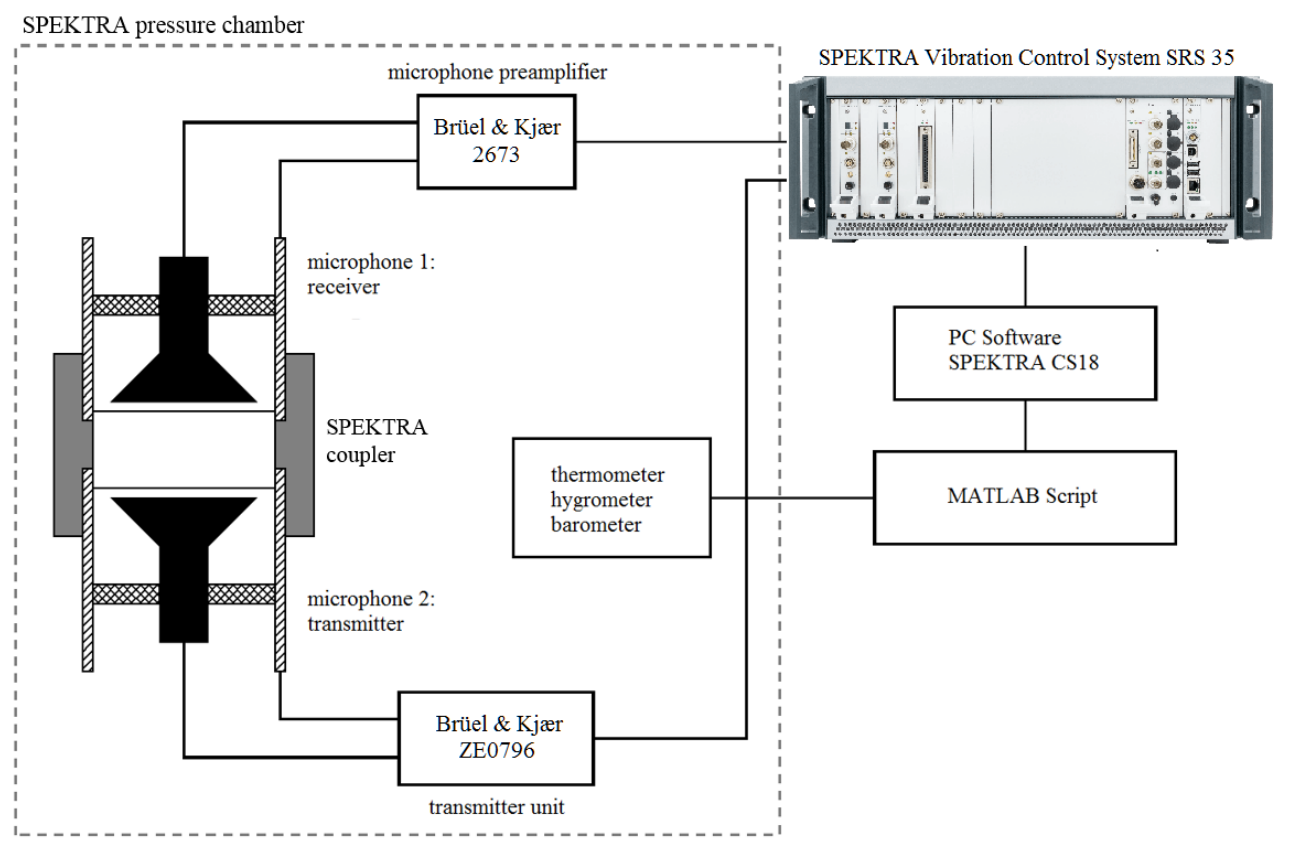

Fig. 1. Schematic diagram of the SPEKTRA reciprocity calibration system

It includes four air-filled sapphire plane-wave couplers, a microphone fixture and a pressure chamber (see Figure 2). 

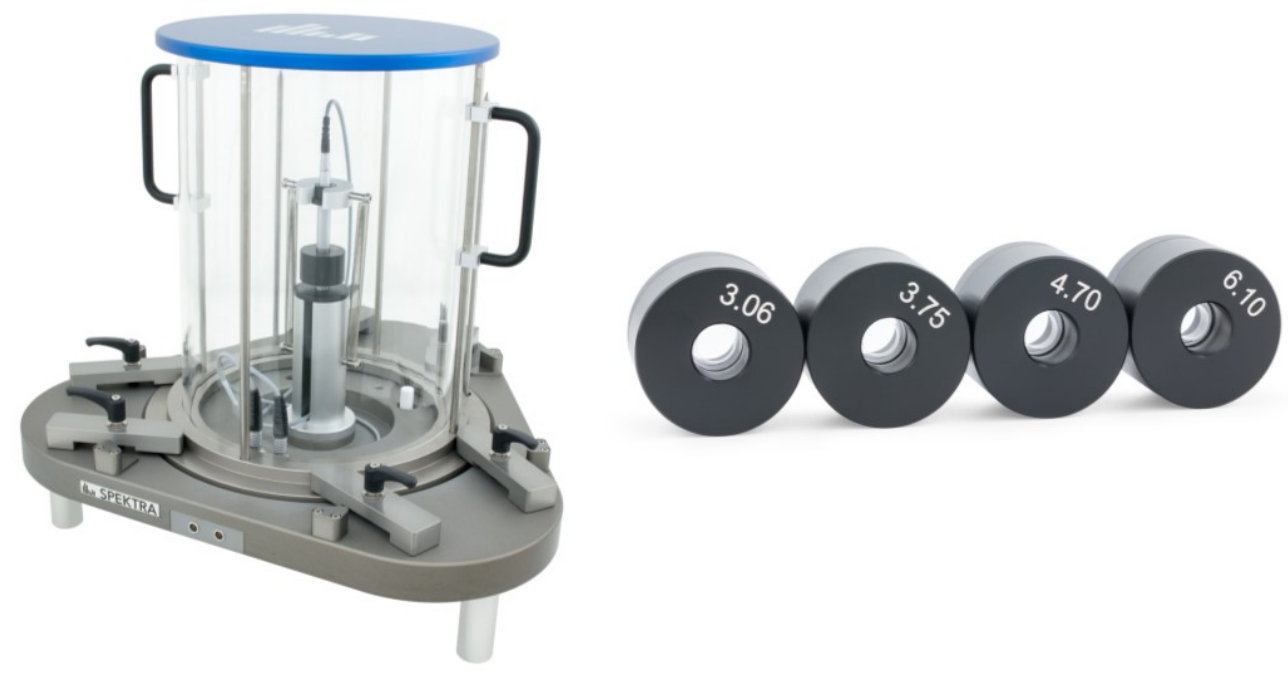

Fig. 2. SPEKTRA pressure chamber (left), SPEKTRA sapphire plane-wave couplers (right)

The microphones are connected to a vibration control system SRS 35 by SPEKTRA via a microphone transmitter unit Brüel \& Kjær ZE0796 and a microphone preamplifier unit Brüel \& Kjær 2673. Furthermore, the software CS18 that controls the SRS 35 and a MATLAB $^{\circledR}$ script for the calculation of the sensitivities are included. The MATLAB ${ }^{\circledR}$ script contains all approaches for determining the sensitivities of the standard [1]. It is possible to compare the approaches and to validate the calculation systematically. The MATLAB $^{\circledR}$ script and the calculation program developed by the Swiss national metrology institute METAS were compared in earlier research. The difference of the calculation is up to $1.3 \cdot 10^{-5} \mathrm{~dB}\left(10^{-6} \mathrm{~dB}\right.$ on the average $)$.

To determine the microphone parameters, SPEKTRA measures the front cavity depth using a laser distance sensor, the reference front cavity diameter value was provided for the manufacturer. Thus the front cavity volume and the equivalent volume have been optimized in a frequency range between $200 \mathrm{~Hz}$ and $2 \mathrm{kHz}$, to minimize the difference between the pressure sensitivities using four couplers according the standard [1]. A reference resonance frequency together with a reference loss factor was determined by a least square fit in a frequency range between $1 \mathrm{kHz}$ and $20 \mathrm{kHz}$. The determination of the microphone parameters can be performed automatically by the MATLAB ${ }^{\circledR}$ script.

\section{Initial investigations}

For the investigation three G.R.A.S. 40AU-1 were evaluated. The G.R.A.S. 40AU-1 is a new version of the G.R.A.S. 40AU. According to the supplier the manufacturing procedures, handling and ageing of the microphones have been improved with respect to the previous model.

The open-circuit sensitivity level of the microphones was determined in a frequency range between $31.5 \mathrm{~Hz}$ and $20 \mathrm{kHz}$. The pressure sensitivities were performed at the reference environmental conditions $\left(23^{\circ} \mathrm{C}\right.$ and $\left.101.325 \mathrm{kPa}\right)$ given in the standard [1]. The results of three measurements within three days of one of the G.R.A.S. 40AU-1 are shown in Figure 3. 


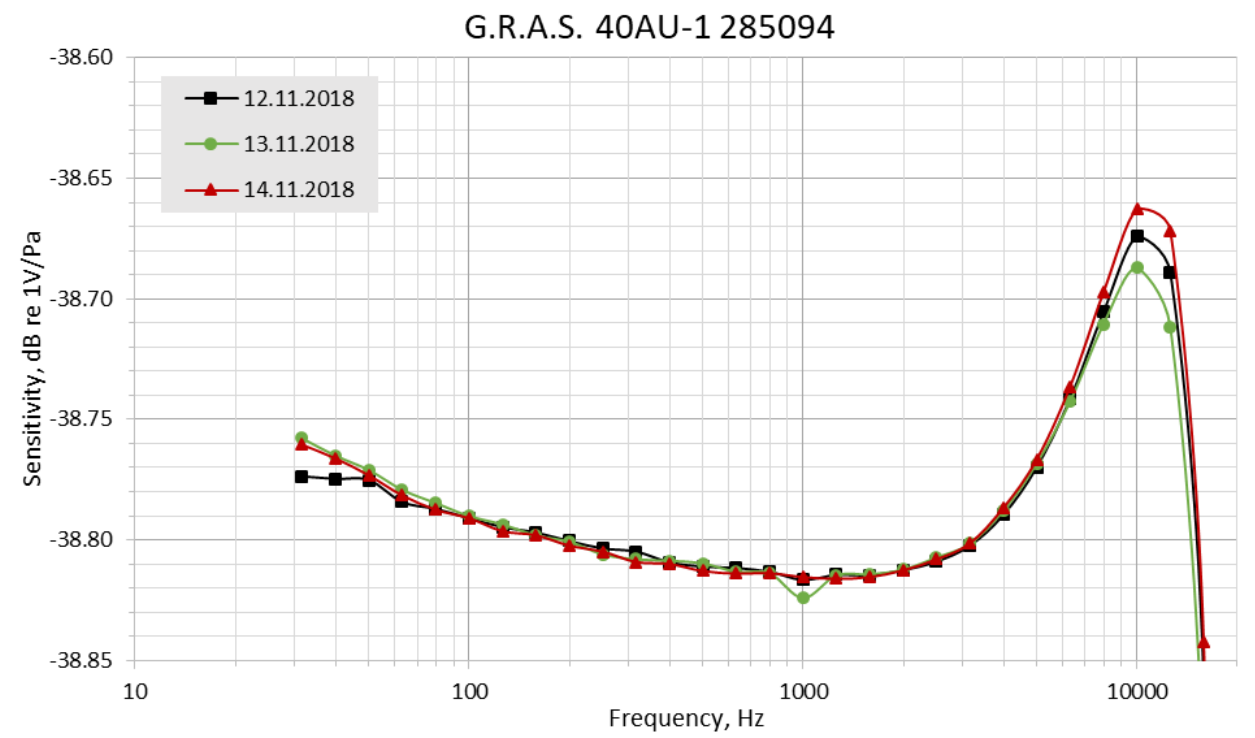

Fig. 3. Pressure sensitivity of G.R.A.S. 40AU-1 285094 determined by SPEKTRA

From these results the short-term stability coefficient of the microphones was approximately calculated. According to the standard [2], it is determined based on the standard deviation of five measurements and must be smaller than $0.02 \mathrm{~dB}$. The average standard deviation for the three measurements is $0.0014 \mathrm{~dB}$ in the required frequency range from $250 \mathrm{~Hz}$ to $1 \mathrm{kHz}$.

Besides the standard microphone combinations, other microphone combinations were measured to estimate the reciprocity of the microphones. Thus the role of the microphones were reversed; the microphone acting as the transmitter in the first step is the receiver in the second step. The deviation between the determined pressure sensitivities should be as small as possible. In this case, the average standard deviation is $0.004 \mathrm{~dB}$.

Furthermore, primary calibrations with one or two G.R.A.S. 40AU-1 and one or two known Brüel \& Kjær 4180 were carried out. These investigations were carried out in cooperation with the Danish Fundamental Metrology DFM, METAS and SPEKTRA. A Brüel \& Kjær 4180 was used as known test standard to verify the calibration method. Figure 4 shows the pressure sensitivity of the microphone Brüel \& Kjær 41802889896 determined by DFM together with two G.R.A.S. 40AU-1, by METAS together with two Brüel \& Kjær 4180 and by SPEKTRA together with one G.R.A.S. 40AU-1 and one Brüel $\&$ Kjær 4180. The sensitivity curves of the microphones obtained when applying the pressure reciprocity technique to such a heterogeneous triplet were consistent with the results obtained when using only microphones of one type. 


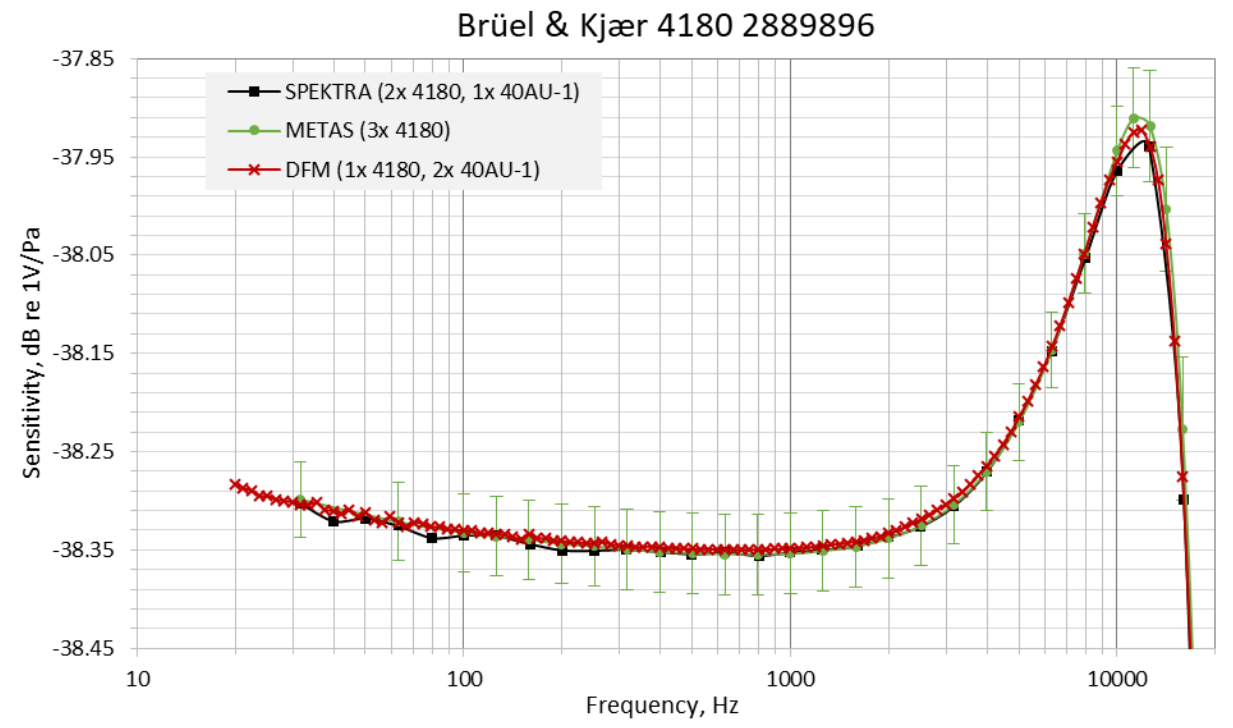

Fig. 4. Pressure sensitivity of Brüel \& Kjær 41802889896 determined by DFM, METAS and SPEKTRA

Finally, the open-circuit sensitivity level of the microphones G.R.A.S. 40AU-1 has been determined over one year. A selection of the results of these measurements for the G.R.A.S. 40AU-1 285094 determined by DFM, METAS and SPEKTRA is shown in Figure 5.

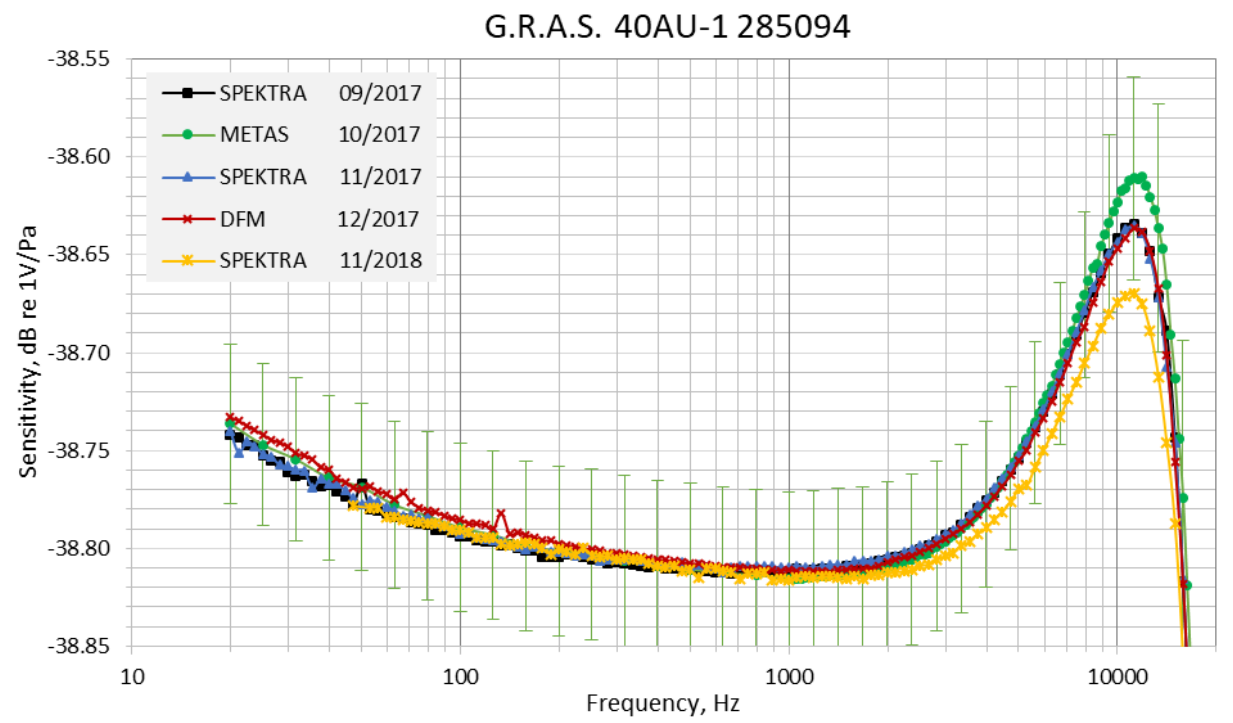

Fig. 5. Pressure sensitivity of G.R.A.S. 40AU-1 285094 determined by DFM, METAS and SPEKTRA

According to the standard [2], the long-term stability coefficient has to be smaller than $0.02 \mathrm{~dB} /$ year. The average standard deviation between the results of the measurements at the end of 2017 and at the end of 2018 is $0.002 \mathrm{~dB}$ in the required frequency range from $250 \mathrm{~Hz}$ to $1 \mathrm{kHz}$.

In the course of these measurements METAS and SPEKTRA also reported the microphone parameters of the G.R.A.S. 40AU-1 285094 (see Table 1). The parameters all 
lie all in the range expected by the standard [2] and by the reports of comparison measurements with $1 / 2$-inch LS microphones.

Table 1. Microphone parameters of G.R.A.S. 40AU-1 285094 determined by METAS, DFM and SPEKTRA

\begin{tabular}{|c|c|c|c|}
\hline & METAS & DFM & SPEKTRA \\
\hline Front cavity volume & $30.87 \mathrm{~mm}^{3}$ & $30.8 \mathrm{~mm}^{3}$ & $32.98 \mathrm{~mm}^{3}$ \\
\hline Front cavity depth & $0.46 \mathrm{~mm}$ & $0.39 \mathrm{~mm}$ & $0.49 \mathrm{~mm}$ \\
\hline Equivalent volume & $9.20 \mathrm{~mm}^{3}$ & $9.0 \mathrm{~mm}^{3}$ & $9.20 \mathrm{~mm}^{3}$ \\
\hline Resonance frequency & $24903 \mathrm{~Hz}$ & $26500 \mathrm{~Hz}$ & $24915 \mathrm{~Hz}$ \\
\hline Loss factor & 1.25 & 1.25 & 1.25 \\
\hline
\end{tabular}

\section{Conclusions}

The initial investigations on the G.R.A.S. 40AU-1 with the new reciprocity calibration system by SPEKTRA provided the basis for further research. It could already be shown that the primary calibration of the G.R.A.S. 40AU-1 is reproducible. Furthermore, it could be shown, that the G.R.A.S. 40AU-1 has a very good short-term stability. The results so far also show a good long-term stability of the G.R.A.S. 40AU-1. Therefore it can be assumed with a high confidence that the targeted measuring accuracy can be achieved with the G.R.A.S. 40AU-1.

Nevertheless, further studies should be conducted to determine the influence of static pressure and temperature on the sensitivity of G.R.A.S. 40AU-1, as has been done for Brüel $\&$ Kjær 4160 and 4180 [3]. Furthermore, the applicability of IEC TS 61094-7 [4] to determine the free-field sensitivity levels for G.R.A.S. 40AU-1 based on the pressure sensitivity levels should be re-examined. Finally, the stability of the G.R.A.S. microphones should be monitored over a longer period. For this purpose further comparison measurements with the German National Metrology Institute PTB, DFM and METAS are planned.

\section{References}

1. International Electrotechnical Commission, IEC 61094-2:2009-Measurement microphones - Part 2: Primary method of pressure calibration of laboratory standard microphones by the reciprocity technique (2009)

2. International Electrotechnical Commission, IEC 61094-1:2000 - Measurement microphones - Part 1: Specifications for laboratory standard microphones (2000)

3. K. Rasmussen, Metrologia, The static pressure and temperature coefficients of laboratory standard microphones, 36 pp 265-273 (1999)

4. International Electrotechnical Commission, IEC TS 61094-7:2006 - Measurement microphones - Part 7: Values for the difference between free-field and pressure sensitivity levels of laboratory standard microphones (2006) 